\title{
Simple heterodyne laser interferometer with subnanometer periodic errors
}

\author{
Ki-Nam Joo, ${ }^{1, *}$ Jonathan D. Ellis, ${ }^{1}$ Jo W. Spronck, ${ }^{1}$ Paul J. M. van Kan, ${ }^{2}$ and Robert H. Munnig Schmidt ${ }^{1}$ \\ ${ }^{1}$ Mechatronic System Design, Department of Precision and Microsystems Engineering, \\ Delft University of Technology, Delft, The Netherlands \\ ${ }^{2}$ NMi Van Swinden Laboratorium BV, Delft, The Netherlands \\ *Corresponding author: k.joo@tudelft.nl
}

Received October 9, 2008; revised December 10, 2008; accepted December 11, 2008; posted January 6, 2009 (Doc. ID 102595); published January 30, 2009

\begin{abstract}
We describe a simple heterodyne laser interferometer that has subnanometer periodic errors and is applicable to industrial fields. Two spatially separated beams can reduce the periodic errors, and the use of a right-angle prism makes the optical configuration much simpler than previous interferometers. Moreover, the optical resolution can be enhanced by a factor of 2 , because the phase change direction is opposite between reference and measurement signals. Experiments have demonstrated the periodic errors are less than $0.15 \mathrm{~nm}$ owing to the frequency mixing of the optical source. The improvements for reducing the frequency mixing of the optical system are also discussed. (C) 2009 Optical Society of America
\end{abstract}

OCIS codes: $120.3180,120.3930,120.3940$.

The periodic errors limit the accuracy of heterodyne laser interferometers because they deteriorate the purity of the interference signals [1]. Since the periodic errors of the laser interferometers were first predicted [2], research pertaining to theoretical models and compensation techniques has been investigated and reported [3-5]. Recently, a real-time first-order periodic error correction technique was validated under various experimental conditions indicating that the remaining periodic error is at the subnanometer level [6]. In addition to compensation methods, nonpolarizing optical configurations to eliminate the periodic errors have been designed [7-9]. The common feature of these interferometers is to use spatially separated beams that have different frequency components. Essentially, the polarization states are never mixed; thus the nonlinearity can be significantly reduced. However, the optical setups are complicated because both beams propagate separately, which requires additional optical components [7]. Although several designs have been reported with heterodyne interferometers using an acousto-optic frequency shifter (AOFS) as a beam splitter [8,9], the small AOFS diffraction angle and their specific configurations limit the applicability for displacement measurements.

In this research, a simple and industry-adaptable heterodyne laser interferometer designed to significantly reduce periodic errors is proposed and tested. The periodic errors are essentially caused by splitting and recombining two nonideal beams using polarizing optics that are both nonideal and are sensitive to alignment. To eliminate the periodic errors in the optical configuration, two beams with different optical frequencies should be spatially separated to avoid the frequency and polarization mixing.

Figure 1 shows the optical configuration that was employed in this investigation. An optical source provides two parallel beams to the interferometer, where each beam has the same polarization state but has different optical frequencies $f_{0}$ and $f_{0}+f_{s}$, respectively.
The two beams propagate to a nonpolarizing beam splitter (NPBS) where they are split into two sets of beams, reference and measurement beams. The reference beams are reflected by a right-angle prism (RAP), and the measurement beams are reflected by a retroreflector $(\mathrm{RR})$. The $\mathrm{RR}$ provides symmetry with respect to a central point and causes the measurement beams to cross each other. The reference beams, on the other hand, have the line symmetry by the RAP. Each set of beams travels back to the NPBS and is recombined to create two beat signals with the frequency of $f_{s}$, which are detected by the photodetectors, $\mathrm{PD}_{1}$ and $\mathrm{PD}_{2}$.

While the RR is moving the measurement beams are phase shifted, caused by the Doppler frequency shift, and are measured by $\mathrm{PD}_{1}$ and $\mathrm{PD}_{2}$. In this case, the heterodyne signals from the photodetectors provide the same amplitude, but the phase shift direction is in the opposite direction between the two signals. Thus the two interference signals from $\mathrm{PD}_{1}$ and $\mathrm{PD}_{2}$ can be expressed by $\cos \left(2 \pi f_{s} t-2 k \Delta L\right)$ and $\cos \left(2 \pi f_{s} t+2 k \Delta L\right)$, respectively, where $k$ is the wavenumber and $\Delta L$ is the displacement of the target $R R$

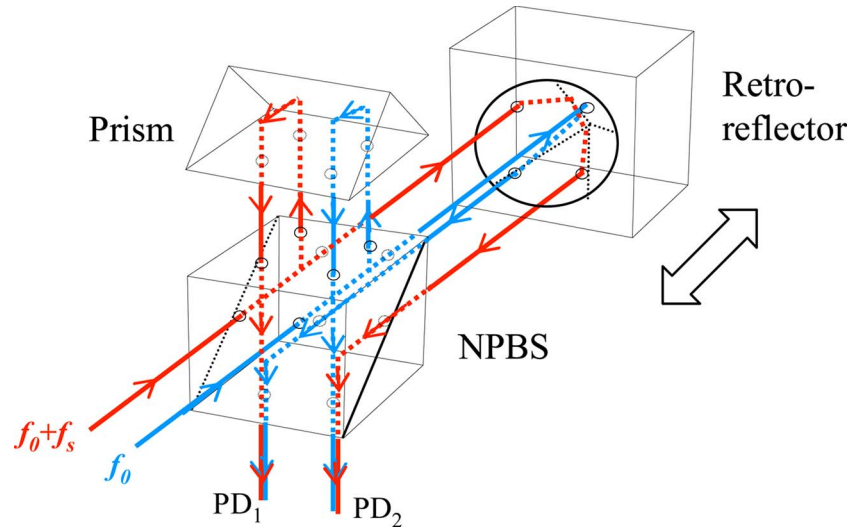

Fig. 1. (Color online) Proposed optical configuration of a heterodyne laser interferometer to reduce the periodic errors. NPBS, nonpolarizing beam splitter; $\mathrm{PD}_{1}, \mathrm{PD}_{2}$, photodetectors. 
while it is moving. When the phase difference between the signals from $\mathrm{PD}_{1}$ and $\mathrm{PD}_{2}$ is measured, the total phase difference is $4 k \Delta L$, which is an effective optical resolution of four. The beams are spatially separated, and the optical paths are not overlapped in the interferometer; therefore no leakage of light is detected except ghost reflections. It should be noted that ghost reflections can be minimized by proper antireflection coatings and alignment techniques. Moreover, polarizing optics, which can generate the frequency and/or polarization mixing, are not used. This interferometer is totally insensitive to the misalignment of optical components, although careful attention is required in the initial alignment owing to the prism.

To validate the effectiveness of the proposed interferometer, we performed feasibility experiments with a commercial heterodyne laser (Axiom 7701, Zygo Corp.) as an optical source, as shown in Fig. 2. The coaxial beams of the heterodyne laser are split into two beams, $f_{1}$ and $f_{2}\left(f_{2}-f_{1}=20 \mathrm{MHz}\right)$, by a polarizing beam splitter (PBS), and the $f_{1}$ beam passes through another AOFS, which induces the frequency shift, $\delta f$, of $19.9 \mathrm{MHz}$. The diffracted beam $\left(f_{1}+\delta f\right)$ and the reflected beam $\left(f_{2}\right)$ by the PBS are adjusted to be parallel by mirrors and have the same polarization state after a polarizer. The final heterodyne frequency split, $f_{2}-\left(f_{1}+\delta f\right)$, is approximately $100 \mathrm{kHz}$. Although the leakage frequency component of each beam is not completely removed from the schematic in Fig. 2, the leakage ratio can be significantly reduced. The two beams can be expressed as

$$
\begin{aligned}
E_{1}= & \exp \left[j\left(2 \pi f_{2} t\right)\right]+\varepsilon_{1} \exp \left[j\left(2 \pi f_{1} t\right],\right. \\
E_{2}= & \exp \left[j\left(2 \pi\left(f_{1}+\delta f\right) t\right]+\varepsilon_{1} \exp \left[j \left(2 \pi \left(f_{2}\right.\right.\right.\right. \\
& +\delta f) t)]+\varepsilon_{2} \exp \left[j\left(2 \pi f_{1} t\right)\right] \\
& +\varepsilon_{1} \varepsilon_{2} \exp \left[j\left(2 \pi f_{2} t\right)\right],
\end{aligned}
$$

where $\varepsilon_{1}$ is the leakage ratio from the laser and the

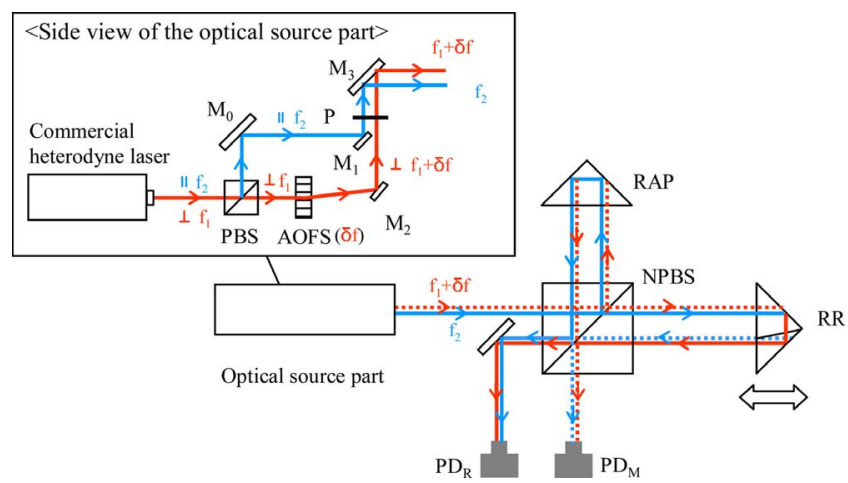

Fig. 2. (Color online) Experimental setup. AOFS, acoustooptic frequency shifter; $\mathrm{M}_{0}, \mathrm{M}_{1}, \mathrm{M}_{2}, \mathrm{M}_{3}$, angle-adjusted mirrors; NPBS, nonpolarizing beam splitter; RAP, rightangle prism; RR, retroreflector; $\mathrm{PD}_{R}, \mathrm{PD}_{M}$, reference and measurement photodetectors; PBS, polarizing beam splitter; $\mathrm{P}$, polarizer. The inlet is the configuration of the optical source with two spatially separated beams on the side view. Note that the solid line is the spatially upper beam, and the dotted line is the lower beam.

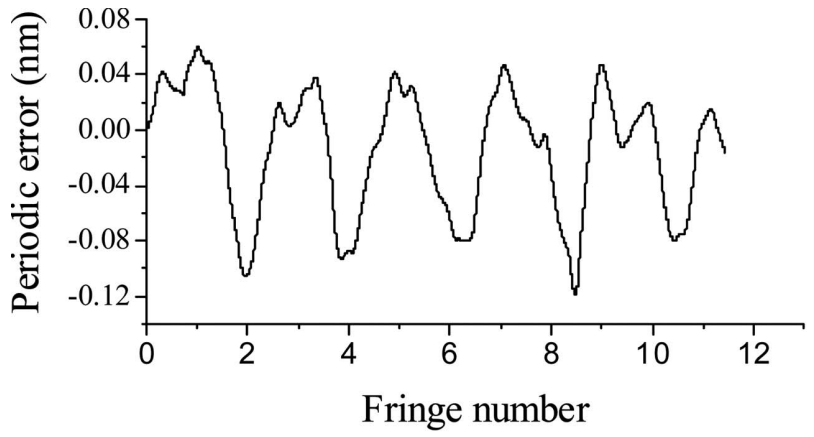

(a)

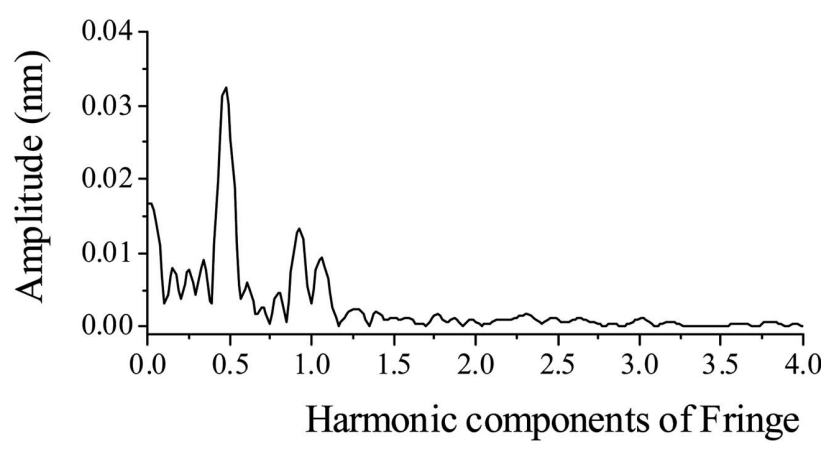

(b)

Fig. 3. Experimental result of the periodic error from the phase quadrature measurement method and (b) Fourier transformed result of the periodic errors (a). The main error is caused by the frequency mixing of the optical source and other noises are from ghost reflections and electronics.

$\mathrm{PBS}$, and $\varepsilon_{2}$ is the leakage ratio of the AOFS. In Eq. (1) and [7], the leakage term causing the phase error is $\varepsilon_{1} \varepsilon_{2} \exp \left[j\left(2 \pi f_{2} t\right)\right]$ and the phase error $(\mathrm{d} \phi)$ and the amplitude change $(\mathrm{d} R / R)$ are expressed from the phase quadrature measurement method in [7] as

$$
\mathrm{d} \phi=2 \varepsilon_{1} \varepsilon_{2} \sin (2 k \Delta L),
$$

$$
\frac{\mathrm{d} R}{R}=2 \varepsilon_{1} \varepsilon_{2} \cos (2 k \Delta L)
$$

From Eq. (2), the phase error is correlated with the amplitude change and the periodic error cycles at half the fringe frequency because the measured phase is $4 k \Delta L$. Figure 3 shows the measurement results of the periodic error in the system and the Fourier transformed result. The amplitude and the phase were measured by a commercial lock-in amplifier (5210, Signal Recovery), while the RR was moved by a piezoelectric stage. The overall periodic errors were calculated using Eq. (2) to be estimated below $0.15 \mathrm{~nm}$ in Fig. 3(a), and the dominant periodic error was caused by the ratios $\varepsilon_{1}$ and $\varepsilon_{2}$ with half the period frequency and the amplitude of $\pm 33 \mathrm{pm}$ in Fig. 3 (b). The sources of remaining peaks with the period frequency in Fig. 3(b) are from the parasitic reflection of the optical components and electrical demodulation noise. 


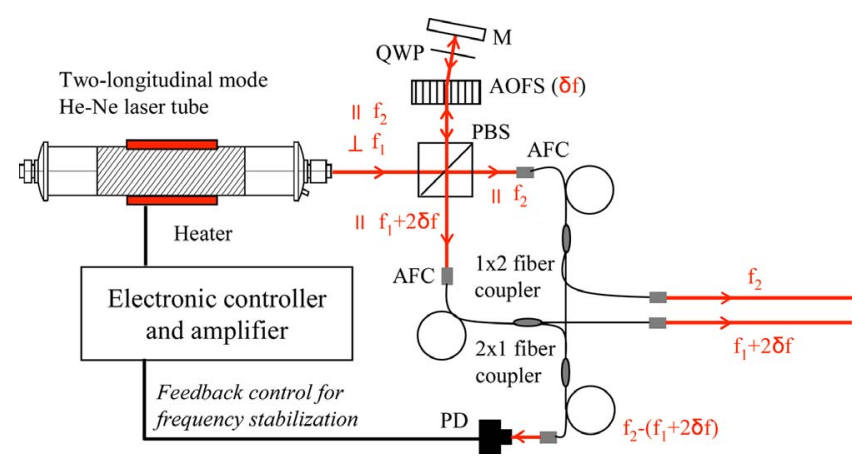

Fig. 4. (Color online) Proposed optical source to obtain a frequency-stabilized laser with two parallel beams to reduce frequency mixing. PBS, polarizing beam splitter; AOFS, acousto-optic frequency shifter; QWP, quarter-wave plate; M, mirror; AFC, angled fiber coupler; $\mathrm{PD}$, photodetector.

To fully verify the theoretical elimination of periodic errors, the laser source must be free from frequency mixing. Employing two AOFSs with different frequency shifts has been shown to remove the frequency mixing [7]. This laser system, however, increases the overall cost, which is not desirable for both research and industrial fields. A frequencystabilized and an offset-locked laser set is another potential alternative to improve the performance of the interferometer, although the phase jitter noise between the two lasers remains [10].

An alternative scheme to obtain two parallel beams with minimal frequency mixing is shown in Fig. 4. The laser source is a two-longitudinal mode $\mathrm{He}-\mathrm{Ne}$ laser tube that has approximately $600 \mathrm{MHz}$ frequency difference, $f_{2}-f_{1}$, and orthogonal polarization states. This initial beam is split into two by a PBS and the reflected beam, $f_{1}$, passes through a polarization sensitive AOFS $(\delta f \sim 300 \mathrm{MHz})$, where the diffracted beam is orthogonally polarized to the input beam. It is reflected by a mirror passing through a quarter-wave-plate (QWP) twice and then back through the AOFS. This beam then passes through the initial PBS and into a fiber coupler. Even though the leakage beam reflected by the PBS goes back to the laser tube, the laser stabilization is not affected owing to the shifted optical frequency [9]. The second beam from the source, $f_{2}$, passes through the initial
PBS and into a fiber coupler. Two fiber splitters are used, and one arm from each set is combined with a second into one fiber coupler to recombine the beams to provide feedback control to obtain a stabilized frequency. The heterodyne frequency is then $f_{2}-\left(f_{1}\right.$ $+2 \delta f$ ), and it is used for frequency stabilization. The orthogonality of the two longitudinal modes, polarization characteristic of the AOFS and passing through the AOFS twice, should reduce the ratio of leakage frequency components.

To summarize, a simple heterodyne laser interferometer was proposed to remove the periodic error inherent to laser interferometers. The reference and measurement beams are spatially separated to prevent any frequency mixing, and the use of a right-angle prism makes it possible to construct a simple interferometer setup and enhance the optical resolution by a factor of 2. Experimental results showed the periodic error to be less than $0.15 \mathrm{~nm}$, which was dominated by the frequency mixing of the optical source. Several options for alternative optical sources to reduce the frequency mixing were also proposed and discussed.

This work was supported by the Dutch Innovatiegerichte Onderzoeksprogramma's (project 04001) and NMi in the Netherlands. The authors thank Eric Buice for his comments on this review.

\section{References}

1. N. Bobroff, Meas. Sci. Technol. 4, 907 (1993).

2. R. C. Quenelle, Hewlett-Packard J. 34, 10 (1983).

3. W. Hou and G. Wilkening, Precis. Eng. 14, 91 (1992).

4. T. Eom, T. Choi, K. Lee, H. Choi, and S. Lee, Meas. Sci. Technol. 13, 222 (2002).

5. S. J. A. G. Cosijns, H. Haitjema, and P. H. J. Schellekens, Precis. Eng. 26, 448 (2002).

6. T. L. Schmitz, D. Chu, and L. Hoouck III, Meas. Sci. Technol. 17, 3195 (2006).

7. C. Wu, J. Lawall, and R. D. Deslattes, Appl. Opt. 38, 4089 (1999).

8. T. L. Schmitz and J. F. Beckwith, J. Mod. Opt. 49, 2105 (2002).

9. J. Lawall and E. Kessler, Rev. Sci. Instrum. 71, 2669 (2000).

10. J. Lawall and J. M. Pedulla, Rev. Sci. Instrum. 72, 2879 (2001). 